\title{
AN ANALOGUE OF A CONJECTURE OF SATO AND TATE FOR A HILBERT MODULAR FORM
}

\author{
by H. L. RESNIKOFF' ${ }^{\dagger}$ and R. L. SALDAÑA
}

(Received 5 April, 1974)

1. Introduction. If $k$ denotes a number field and $\varepsilon^{m}$ is the product of an elliptic curve $\varepsilon$ with itself $m$ times over $k$, then for each prime $\pi$ where $\varepsilon$ has non-degenerate reduction, the zeta factor $\zeta\left(\varepsilon_{n}, s\right)$ can be expressed as

$$
\zeta\left(\varepsilon_{\pi}, s\right)=\frac{\left(1-\varepsilon_{\pi}|\pi|^{\frac{1}{2}-s}\right)\left(1-\bar{\varepsilon}_{\pi}|\pi|^{\frac{1}{2}-s}\right)}{\left(1-|\pi|^{-s}\right)\left(1-|\pi|^{1-s}\right)},
$$

where $|\pi|$ denotes the norm of $\pi$. It is a consequence of a conjecture of Tate [16] that if $\varepsilon$ does not have complex multiplications, then the numbers $x_{\pi}=\frac{1}{2}\left(\varepsilon_{\pi}+\bar{\varepsilon}_{\pi}\right)$ are distributed according to the density function

$$
\frac{2}{\pi}\left(1-x^{2}\right)^{\frac{1}{2}}
$$

that is, the density of the set of primes $\pi$ such that $-1 \leqq a \leqq \frac{1}{2}\left(\varepsilon_{\pi}+\bar{\varepsilon}_{\pi}\right) \leqq b \leqq 1$ is

$$
\frac{2}{\pi} \int_{a}^{b}\left(1-x^{2}\right)^{\frac{1}{2}} d x .^{\ddagger}
$$

According to Tate [16], machine calculations conducted by M. Sato support this conclusion.

Serre [13] (cf. [14], [15]) posed an analogous question for Ramanujan's function $\tau$. Put

$$
\Delta(z)=e^{2 \pi i z}\left\{\prod_{n=1}^{\infty}\left(1-e^{2 \pi i n z}\right)\right\}^{24}, \operatorname{Im} z>0 .
$$

$\Delta$ is a cusp form of weight 12 for the classical modular group $S L(2, \mathbb{Z}) /\{ \pm I d\}$, hence an eigenfunction of the ring of Hecke operators. If the Fourier expansion of $\Delta$ be written

$$
\Delta(z)=\sum_{n=1}^{\infty} \tau(n) e^{2 \pi i n z},
$$

then $\tau(1)=1$, the function $\tau: \mathbb{Z}^{+} \rightarrow R$, which is Ramanujan's function, is multiplicative, and the associated Dirichlet series

$$
\zeta_{\Delta}(s)=\sum_{n=1}^{\infty} \tau(n) n^{-s}
$$

† Supported in part by National Science Foundation grant GP19011.

$\ddagger$ The reader is not likely to confuse use of the common symbol $\pi$ to denote primes in a number field and the ratio of circumference to diameter of a circle. 
admits the Euler product expansion

$$
\zeta_{\Delta}(s)=\prod_{p}\left(1-\tau(p) p^{-s}+p^{11-2 s}\right)^{-1}
$$

where $p$ runs through the rational primes. A celebrated conjecture of Ramanujan [10] asserted that

$$
\text { abs } \tau(p) \leqq 2 p^{11 / 2},
$$

where $a b s$ denotes the absolute value. Deligne [2] showed that (3) is a consequence of Weil's conjectures, and Deligne [3] has recently proved the latter; hence (3) is valid and therefore, writing $x_{p}=\tau(p) / 2 p^{11 / 2}$, one has $-1 \leqq x_{p} \leqq 1$ and the factor $\left(1-\tau(p) p^{-s}+p^{11-2 s}\right)$ in $(2)$ can be written as $\left(1-\varepsilon_{p} p^{11 / 2-s}\right)\left(1-\bar{\varepsilon}_{p} p^{11 / 2-s}\right)$, where $a b s \quad \varepsilon_{p}=1$ and $2 x_{p}=\varepsilon_{p}+\bar{\varepsilon}_{p}$. Serre's question $\left([13]\right.$, pp. 14-15) is whether $x_{p}$ is also distributed according to (1) as $p$ runs through the rational primes. Lehmer [9] calculated the distribution of $x_{p}$ for the 1229 primes less than $10^{4}$ and found, in his words, "pretty reassuring" agreement with the Sato-Tate distribution (1).

The principal purpose of this paper is to provide numerical evidence which supports the Sato-Tate distribution for an analogue of Serre's question for Hilbert modular forms. The graded ring of Hilbert modular forms associated with the number field $\mathbb{Q}(\sqrt{ } 5)$ is generated by an Eisenstein series of weight 2, two cusp forms of respective weights 6 and 15 which are unchanged when the argument variables are interchanged, and a "skew" cusp form $\chi_{5}$ of weight 5 which changes sign when its argument variables are interchanged [4], [11]. Since the dimension of the $\mathbb{C}$-linear space of cusp forms of weight 5 is $1, \chi_{5}$ is an eigenfunction of the ring of Hecke operators associated with the Hilbert modular group [7], and it follows that the Fourier coefficients of a suitably normalized $\chi_{5}$ are multiplicative functions of the algebraic integers in $\mathbb{Q}(\sqrt{ } 5)$. Write the Fourier expansion of $\chi_{5}$ in the form

$$
\chi_{5}(\check{\zeta})=\sum c(v) e^{2 \pi i a(v \zeta / \sqrt{ } 5)}
$$

where $\zeta=\left(\zeta_{1}, \zeta_{2}\right), \operatorname{Im} \zeta_{i}>0, \sigma$ denotes the trace (cf. $\left.\S 2\right)$ and $v$ runs through those integers in $\mathbb{Q}(\sqrt{ } 5)$ such that $v / \sqrt{ } 5$ is totally positive.

Select $\varepsilon=(1+\sqrt{ } 5) / 2$ as fundamental unit of $\mathbb{Q}(\sqrt{ } 5)$, and introduce the "Grössencharakter"

$$
\lambda(\mu)=\exp \left\{\frac{i \pi}{4} \sigma\left(\frac{\log a b s \mu}{\log a b s \varepsilon}\right)\right\} \text { for } \mu \in \mathbb{Q}(\sqrt{ } 5),
$$

where the principal branch of the logarithm is chosen. A Dirichlet series associated with $\chi_{5}$ is

$$
\zeta_{\chi_{s}}(s, \lambda)=\sum_{(v)}-i c(v) \lambda(v) a b s|v|^{-s},
$$

where the sum runs over the ideals in the ring of integers in $\mathbb{Q}(\sqrt{ } 5)$; it is readily checked that the individual summands are independent of the choice of representative generator $v$ of $(v)$. A special case of a remarkable theorem of Hermann [7] asserts that $\zeta_{x s}$ can be expressed by the Euler product

$$
\zeta_{\chi s}(s, \lambda)=\prod_{(\pi)}\left(1+i c(\pi) \lambda(\pi) a b s|\pi|^{-s}+a b s|\pi|^{4-2 s}\right)^{-1}
$$


if $\chi_{s}$ is so normalized that $c(\varepsilon)=1$ (the product runs over the prime ideals in the ring of integers of $\mathbb{Q}(\sqrt{ } 5)$ ). Moreover, $\zeta_{x_{5}}$ satisfies the functional equation (Hermann [7], Satz 15):

$$
G(5-s, \bar{\lambda})=-G(s, \lambda)
$$

where

$$
G(s, \lambda)=\left(\frac{5}{4 \pi^{2}}\right)^{s} \Gamma\left(s-\frac{i \pi}{4 \log a b s|\varepsilon|}\right) \Gamma\left(s+\frac{i \pi}{4 \log a b s|\varepsilon|}\right) \zeta_{\chi_{s}}(s, \lambda)
$$

Now suppose that the following analogue of Petersson's extension of Ramanujan's conjecture is valid: if $a(v)$ denotes the $v$ th Fourier coefficient of a normalized cusp form of weight $w$ for Hilbert's modular group associated with some number field, then for prime ideals $\pi$,

$$
a b s a(\pi) \leqq 2(a b s|\pi|)^{(w-1) / 2} .
$$

For $\chi_{5}$ this becomes

$$
a b s c(\pi) \leqq 2(a b s|\pi|)^{2} \text {. }
$$

Set

$$
x_{(\pi)}=c(\pi) / 2(a b s|\pi|)^{2}
$$

then (8) implies that $-1 \leqq x_{(\pi)} \leqq 1$ and, in analogy to the above for the classical modular form $\Delta$, the factor $\left(1+i c(\pi) \lambda(\pi) a b s|\pi|^{-s}+a b s|\pi|^{4-2 s}\right)$ can be written as $\left(1-\varepsilon_{(\pi)} a b s|\pi|^{2-s}\right)$ $\left(1-\bar{\varepsilon}_{(\pi)} a b s|\pi|^{2-s}\right)$ where $a b s \varepsilon_{(\pi)}=1$ and $(-i \lambda(\pi)) x_{\pi}=\left(\varepsilon_{(\pi)}+\bar{\varepsilon}_{(\pi)}\right) / 2$. We are therefore led to inquire whether the $x_{(\pi)}$ are distributed according to the Sato-Tate distribution (1) as $(\pi)$ runs through the prime ideals. The numerical evidence presented in the next section strongly suggests an affirmative answer.

The authors thank F. J. Dyson for bringing Tate's conjecture to our attention. We are indebted to J.-P. Serre for many helpful remarks and particularly for informing us of certain omissions in a previous version of the work reported here.

The remainder of this paper is organized as follows. Section 2 presents a table of the Fourier coefficients $c(\pi)$ of $\chi_{5}$ for the 305 prime ideals of norm $\leqq 4391$, and also shows how the $c(v)$ can be expressed in terms of $c(\pi)$ for arbitrary integral ideals $(v)$; this interdependence provides a check on the validity of the tabulated entries. Section 2 also presents several statistical measures of the compatibility of the distribution of the $x_{(\pi)}$ for the tabulated values with the Sato-Tate distribution. Calculation of the coefficients $c(v)$ is not a straightforward matter. Section 3 describes a connection between Siegel modular forms of degree two and Hilbert modular forms associated with certain quadratic number fields which enables the Fourier coefficients of particular Hilbert modular forms to be expressed as linear combinations of the Fourier coefficients of Siegel modular forms. Specialization of this relationship yields the formula by means of which the values tabulated in $\$ 2$ were calculated. Section 4 applies some of the results of $\S 3$ to Hilbert modular forms associated with quadratic fields other than $\mathbb{Q}(\sqrt{ } 5)$. 
2. The Fourier coefficients of $\chi_{5}$. Define a multiplication on $\mathbb{C}^{2}$ by the bilinear pairing $(\alpha, \beta)=\left(\left(\alpha_{1}, \alpha_{2}\right),\left(\beta_{1}, \beta_{2}\right)\right) \mapsto\left(\alpha_{1} \beta_{1}, \alpha_{2} \beta_{2}\right)=\alpha \beta$ and introduce the trace $\sigma(\alpha)=\alpha_{1}+\alpha_{2}$ and the norm $|\alpha|=\alpha_{1} \alpha_{2}$ for $\alpha \in \mathbb{C}^{2}$. If $k$ is a real quadratic number field and $\alpha_{1}, \alpha_{2}=\alpha_{1}^{*}$ are conjugates in $k$, put $\alpha=\left(\alpha_{1}, \alpha_{2}\right) \in \mathbb{R}^{2} \subset \mathbb{C}^{2}$; in this way $k$ is embedded in $\mathbb{C}^{2}$ and the restriction to the image of $k$ in $\mathbb{C}^{2}$ of the trace and norm defined above are, respectively, the trace and norm in $k$. Extend the conjugation map to $\mathbb{C}^{2}$ by defining ${ }^{*} \alpha={ }^{*}\left(\alpha_{1}, \alpha_{2}\right)=\left(\alpha_{2}, \alpha_{1}\right)=\alpha^{*}$ for $\alpha \in \mathbb{C}^{2}$. If $\alpha \in \mathbb{R}^{2}$, write $\alpha>0$ if $\alpha_{1}>0, \alpha_{2}>0$, and set $\mathscr{H}=\left\{\zeta \in \mathbb{C}^{2}: \operatorname{Im} \zeta>0\right\}$.

Let $k=\mathbb{Q}(\sqrt{ } 5)$ and introduce the fundamental unit $\varepsilon_{1}=(1+\sqrt{ } 5) / 2$; set $\varepsilon=\left(\varepsilon_{1}, \varepsilon_{1}^{*}\right)$. Since $\chi_{5}$ is a skew Hilbert modular form of weight $5, \chi_{5}\left(\varepsilon^{2 n} \zeta\right)=(-)^{n} \chi_{5}(\zeta)$ and $\chi_{5}\left(\zeta^{*}\right)=$ $-\chi_{5}(\zeta)$ for $\zeta \in \mathscr{H}$; these conditions are equivalent to the following two conditions for the Fourier coefficients $c(v)$ of $\chi_{s}$ :

$$
c\left(\varepsilon^{2 n} v\right)=(-)^{n} c(v)
$$

and

$$
c\left(-v^{*}\right)=-c(v)
$$

These equations have the consequence that it is only necessary to calculate the $c(v)$ for those $v$ which lie in a fundamental domain for the action of the group $\Xi^{*}$ generated by the transformations $\eta \mapsto \varepsilon^{2} \eta$ and $\eta \mapsto-\eta^{*}$ on the set of $\eta \in \mathbb{R}^{2}$ such that $\eta / \sqrt{ } 5>0$. An element $\eta \in \mathbb{R}^{2}$ such that $\eta / \sqrt{ } 5>0$ is said to be reduced with respect to the group of units if

$$
\frac{\varepsilon_{2}}{\varepsilon_{1}}>\frac{\eta_{1}}{\eta_{2}} \geqq \frac{\varepsilon_{1}}{\varepsilon_{2}} ;
$$

thus $\varepsilon$ itself is reduced with respect to the group of units. The set $\mathfrak{F}_{\varepsilon}$ of elements reduced with respect to the group of units is easily seen to be a fundamental domain for the action of the group of units defined above. Furthermore, $\mathscr{F}_{*}=\left\{\eta: \eta_{2} \geqq \eta_{1}\right.$ and $\left.\eta / \sqrt{ } 5>0\right\}$ is a fundamental domain for the involution $\eta \mapsto-\eta^{*}$. Then

$$
\mathfrak{F}_{e} \cap \mathfrak{F}_{*} \equiv \mathfrak{F}=\left\{\eta:-1 \geqq \frac{\eta_{1}}{\eta_{2}} \geqq \frac{\varepsilon_{1}}{\varepsilon_{2}} \text { and } \frac{\eta}{\sqrt{ } 5}>0\right\}
$$

is a fundamental domain for the jointly generated group $\Xi^{*}$. Elements of $\mathfrak{F}$ will be said to be $\Xi^{*}$-reduced; $c(v)$ can be calculated from $(9)$ and $(10)$ if $c(\mu)$ is known, where $\mu$ is reduced and equivalent to $v$ under the action of $\Xi^{*}$.

If $\pi$ is a prime in $\mathbb{Q}(\sqrt{ } 5)$, then there is a bijective correspondence between $a b s|\pi|$ and the $\Xi^{*}$-reduced element of the $\Xi^{*}$-orbit of $\pi / \sqrt{ } 5$; we may therefore write $c(a b s|\pi|)$ to unambiguously denote $c(\tilde{\pi})$ where $\tilde{\pi}$ is the $\Xi^{*}$-reduced element of the $\Xi^{*}$-orbit of $\pi / \sqrt{ } 5$ and $\pi / \sqrt{ } 5>0$. The reader will remark that this bijective correspondence cannot be extended to norms of non prime integers of $\mathbb{Q}(\sqrt{ } 5)$; for instance, $a b s\left|v v^{*}\right|=a b s\left|v^{2}\right|$ but $v^{2}$ and $v v^{*}$ need not belong to the same $\Xi^{*}$-orbit.

The absolute values of the norms of primes, that is the norms of prime ideals $(\pi) \subset \mathbb{Q}(\sqrt{ } 5)$, are those primes congruent to 1 or $9 \bmod 10$, those squares of primes congruent to 3 or 7 
$\bmod 10$, and 5 , according as the norm is of the form abs $\pi \pi^{*}$ with $(\pi) \neq\left(\pi^{*}\right), \pi$ is a rational prime, or $(\pi)=\left(\pi^{*}\right)$ respectively. Table I below lists the 305 Fourier coefficients $c(\pi)$ in the form $c(q)$ where $q=a b s|\pi| \leqq 4391$, for primes $\pi \in \mathbb{Q}(\sqrt{ } 5)$ such that $\pi / \sqrt{ } 5$ is totally real and $\Xi^{*}$-reduced.

TABle I. Fourier COEFficients of the Normalized Cusp Form of Weight 5 for Hilbert's Modular Group Associated with $\mathbb{Q}\left(5^{\ddagger}\right)$ for Reduced Prime Classes and for the Reduced UNIT Class

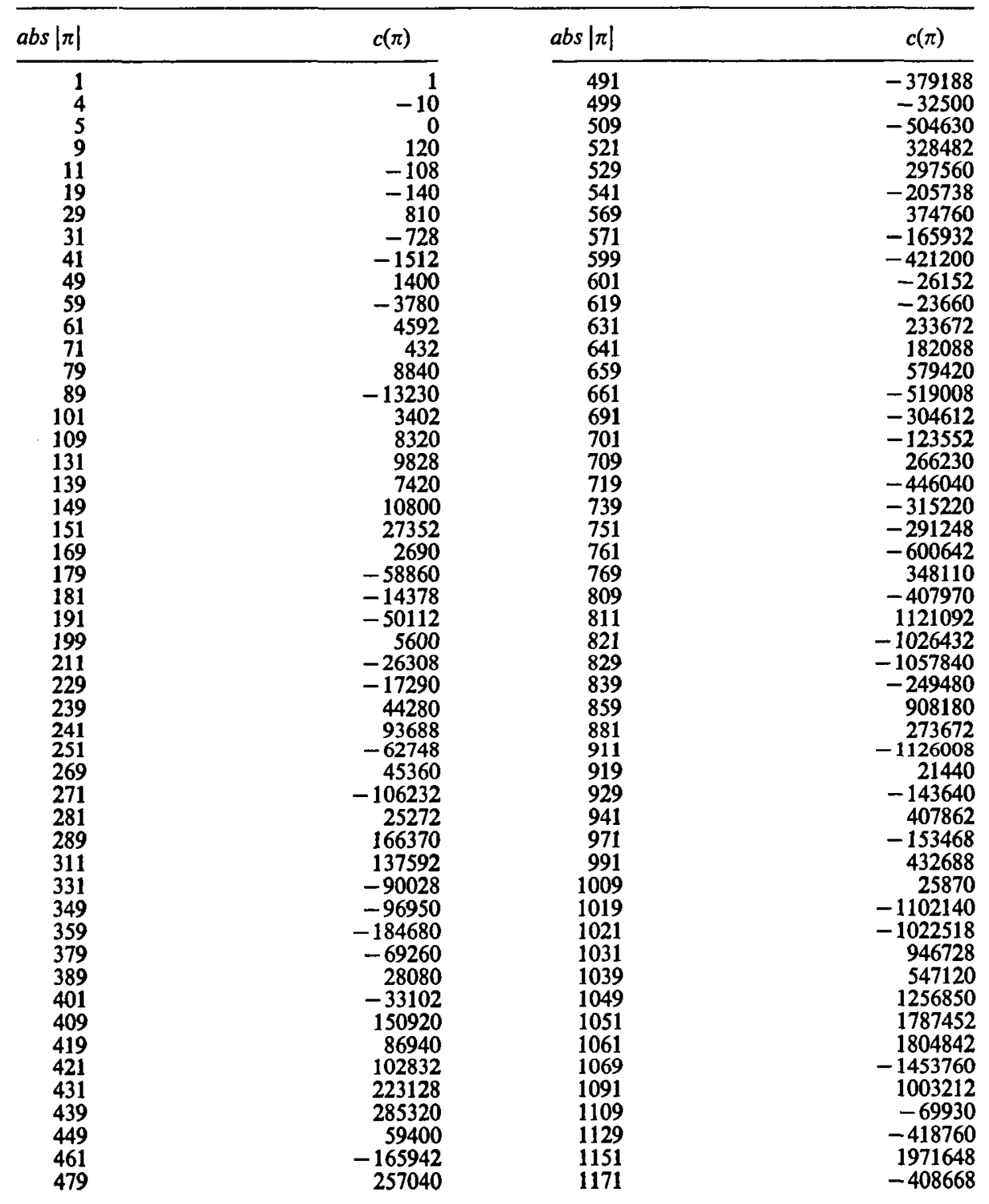


TABLE I-Continued

\begin{tabular}{|c|c|c|c|}
\hline$a b s|\pi|$ & $c(\pi)$ & $a b s|\pi|$ & $c(\pi)$ \\
\hline $\begin{array}{l}1181 \\
1201 \\
1229 \\
1231 \\
1249 \\
1259 \\
1279 \\
1289 \\
1291 \\
1301 \\
1319 \\
1321 \\
1361 \\
1369 \\
1381 \\
1399 \\
1409 \\
1429 \\
1439 \\
1451 \\
1459 \\
1471 \\
1481 \\
1489 \\
1499 \\
1511 \\
1531 \\
1549 \\
1559 \\
1571 \\
1579 \\
1601 \\
1609 \\
1619 \\
1621 \\
1669 \\
1699 \\
1709 \\
1721 \\
1741 \\
1759 \\
1789 \\
1801 \\
1811 \\
1831 \\
1849 \\
1861 \\
1871 \\
1879 \\
1889 \\
1901 \\
1931 \\
1949 \\
1951 \\
1979 \\
1999 \\
2011 \\
2029 \\
2039 \\
2069\end{array}$ & $\begin{array}{r}1276128 \\
-2307448 \\
-2113290 \\
-2305072 \\
-2889250 \\
2566620 \\
-833560 \\
-2259630 \\
-1492988 \\
1140048 \\
-347760 \\
-1825432 \\
-2593458 \\
2877410 \\
1196672 \\
1773800 \\
3274830 \\
1699360 \\
-1597320 \\
-753948 \\
-1532020 \\
-179968 \\
306072 \\
504280 \\
1849500 \\
-2632392 \\
-11228 \\
1628150 \\
4256280 \\
4198068 \\
1027660 \\
-2453598 \\
3197320 \\
-2495340 \\
-3843382 \\
1555190 \\
3607100 \\
-1754730 \\
-555282 \\
-1712438 \\
-968120 \\
1928630 \\
-3562952 \\
-2689092 \\
1581472 \\
6834200 \\
2579542 \\
2311632 \\
1472240 \\
52920 \\
-189648 \\
-5055372 \\
-2948400 \\
-5921552 \\
-5885460 \\
-3566000 \\
4330108 \\
4831190 \\
-4146120 \\
279990\end{array}$ & $\begin{array}{l}2081 \\
2089 \\
2099 \\
2111 \\
2129 \\
2131 \\
2141 \\
2161 \\
2179 \\
2209 \\
2221 \\
2239 \\
2251 \\
2269 \\
2281 \\
2309 \\
2311 \\
2339 \\
2341 \\
2351 \\
2371 \\
2381 \\
2389 \\
2399 \\
2411 \\
2441 \\
2459 \\
2521 \\
2531 \\
2539 \\
2549 \\
2551 \\
2579 \\
2591 \\
2609 \\
2621 \\
2659 \\
2671 \\
2689 \\
2699 \\
2711 \\
2719 \\
2729 \\
2731 \\
2741 \\
2749 \\
2789 \\
2791 \\
2801 \\
2809 \\
2819 \\
2851 \\
2861 \\
2879 \\
2909 \\
2939 \\
2969 \\
2971 \\
2999 \\
3001\end{array}$ & $\begin{array}{r}7889778 \\
-1917230 \\
1568700 \\
558792 \\
543240 \\
646828 \\
-4043088 \\
4796008 \\
5579860 \\
9695480 \\
-6242032 \\
4780720 \\
-1563748 \\
-8527360 \\
-42728 \\
1602720 \\
3019592 \\
-1411020 \\
5017712 \\
-3634848 \\
-2680132 \\
6395328 \\
-3855830 \\
-10168200 \\
7905492 \\
4222638 \\
937980 \\
-2159768 \\
1874772 \\
-9041620 \\
7581600 \\
5837048 \\
-6603660 \\
9918288 \\
-7915320 \\
-9135882 \\
12907580 \\
13555168 \\
-8042680 \\
7055100 \\
-6239808 \\
13725040 \\
1302210 \\
2145572 \\
9680688 \\
-11606000 \\
-9087120 \\
-4740512 \\
-2550798 \\
-3412030 \\
-502740 \\
142652 \\
5911542 \\
6483240 \\
10450080 \\
-3519180 \\
6303960 \\
-2876468 \\
3780000 \\
-10270498\end{array}$ \\
\hline
\end{tabular}


TABLE I-Continued

\begin{tabular}{|c|c|c|c|}
\hline$a b s|\pi|$ & $c(\pi)$ & $a b s|\pi|$ & $c(\pi)$ \\
\hline $\begin{array}{l}3011 \\
3019 \\
3041 \\
3049 \\
3061 \\
3079 \\
3089 \\
3109 \\
3119 \\
3121 \\
3169 \\
3181 \\
3191 \\
3209 \\
3221 \\
3229 \\
3251 \\
3259 \\
3271 \\
3299 \\
3301 \\
3319 \\
3329 \\
3331 \\
3359 \\
3361 \\
3371 \\
3389 \\
3391 \\
3449 \\
3461 \\
3469 \\
3491 \\
3499 \\
3511 \\
3529 \\
3539 \\
3541 \\
3559 \\
3571 \\
3581 \\
3631 \\
3659 \\
3671 \\
3691 \\
3701 \\
3709 \\
3719 \\
3739 \\
3761 \\
3769 \\
3779 \\
3821\end{array}$ & $\begin{array}{r}13931892 \\
11381140 \\
-11341512 \\
-5711150 \\
-12755408 \\
9204160 \\
-2537730 \\
-10265680 \\
6261840 \\
-2020382 \\
3757810 \\
-6563872 \\
5025888 \\
-10455480 \\
-18067968 \\
-5432960 \\
-1385748 \\
-11894620 \\
15234232 \\
12428100 \\
-19591702 \\
-13575760 \\
-5408910 \\
-1280972 \\
-7960680 \\
-4417208 \\
-6422868 \\
-3720330 \\
-235088 \\
-5556600 \\
-13490442 \\
-3150710 \\
-9961812 \\
21668500 \\
3707392 \\
14734190 \\
-23407380 \\
-16595488 \\
-7434280 \\
12551068 \\
17827722 \\
-21644672 \\
13755420 \\
-11361168 \\
15754388 \\
-17696448 \\
-14947520 \\
-76404960 \\
22404060 \\
14560182\end{array}$ & $\begin{array}{l}3851 \\
3881 \\
3889 \\
3911 \\
3919 \\
3929 \\
3931 \\
3989 \\
4001 \\
4019 \\
4021 \\
4049 \\
4051 \\
4079 \\
4091 \\
4099 \\
4111 \\
4129 \\
4139 \\
4159 \\
4201 \\
4211 \\
4219 \\
4229 \\
4231 \\
4241 \\
4259 \\
4261 \\
4271 \\
4289 \\
4339 \\
4349 \\
4391\end{array}$ & $\begin{array}{r}-3776652 \\
4195422 \\
167080 \\
13858992 \\
14889560 \\
-5509890 \\
-24795628 \\
-6571530 \\
21233502 \\
4720140 \\
-17402518 \\
9799650 \\
-18743452 \\
-16548840 \\
1831788 \\
15955300 \\
-14608208 \\
-26422760 \\
19859580 \\
-13959920 \\
19793198 \\
18133308 \\
-23343460 \\
-27560790 \\
14947072 \\
4008312 \\
33562620 \\
-63392 \\
-10622232 \\
1449630 \\
-32613980 \\
-19666800 \\
6527088\end{array}$ \\
\hline
\end{tabular}

We have calculated the $x_{(\pi)}$ which correspond to the tabulated values and tested the Sato-Tate conjecture in several ways. The first four moments of the distribution (1) and the corresponding values obtained from the 305 values in Table I are: 
TABLE II

\begin{tabular}{ccc}
\hline Moment & Distribution (1) & Data from Table I \\
\hline 1 & 0 & -0.0106 \\
2 & $1 / 4=0.25$ & 0.2494 \\
3 & 0 & 0.0088 \\
4 & $1 / 8=0.125$ & 0.1231 \\
\hline
\end{tabular}

The value of chi-squared for 19 degrees of freedom is 14.61. Moreover, application of the Kolmogorov-Smirnov test leads to Figure 1. These three tests each signal a high degree of compatibility of the tabulated data with the distribution (1). Figure 2 displays a histogram of the $\chi_{(\pi)}$-distribution and the Sato-Tate distribution which it approximates.

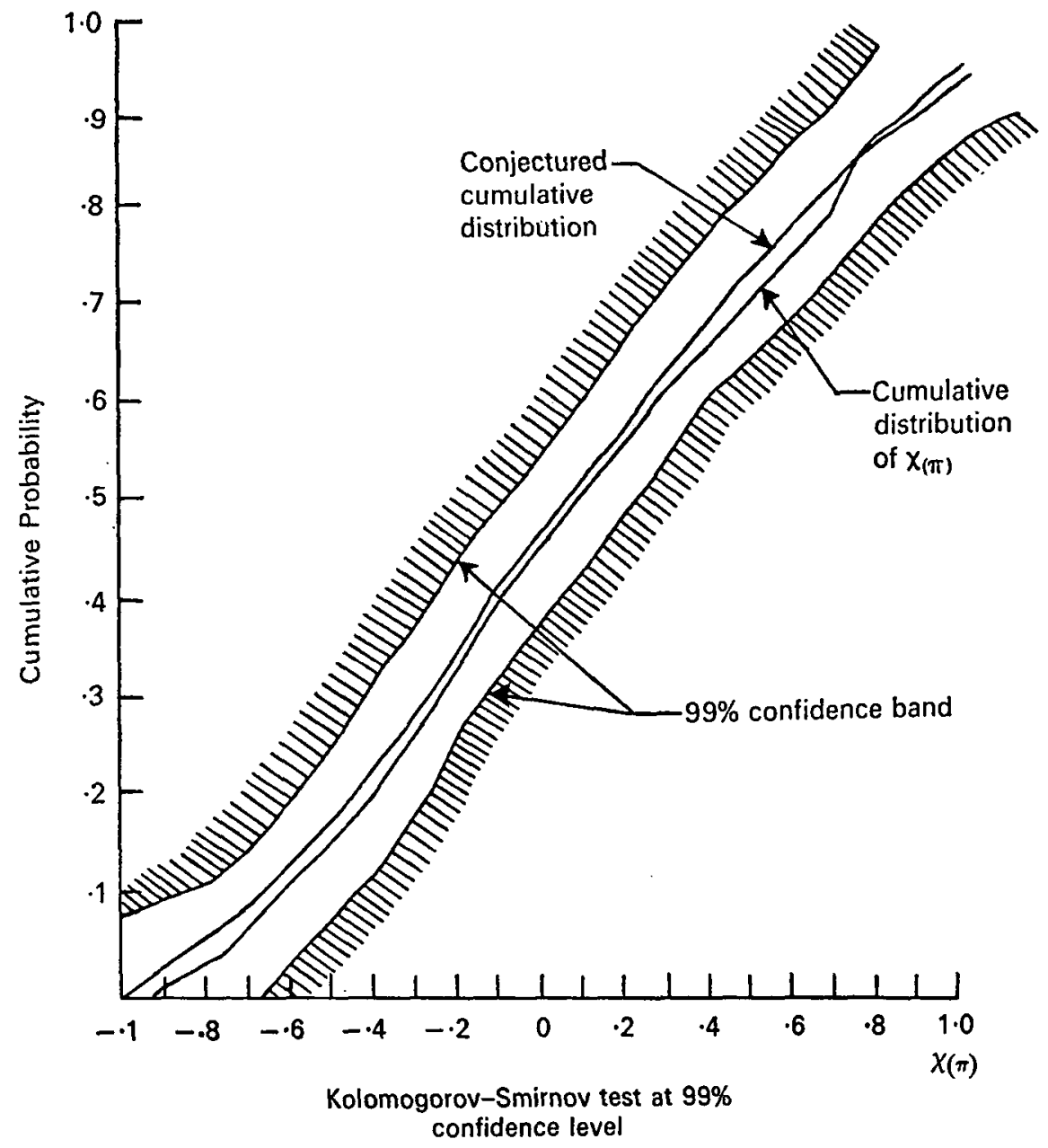

FIGURE 1 
The reader will naturally want to know how the numbers in Table I were calculated, and what checks were employed to verify that they are correct. The first question will be answered in $§ 3$; some brief remarks concerning the second question will be made here.

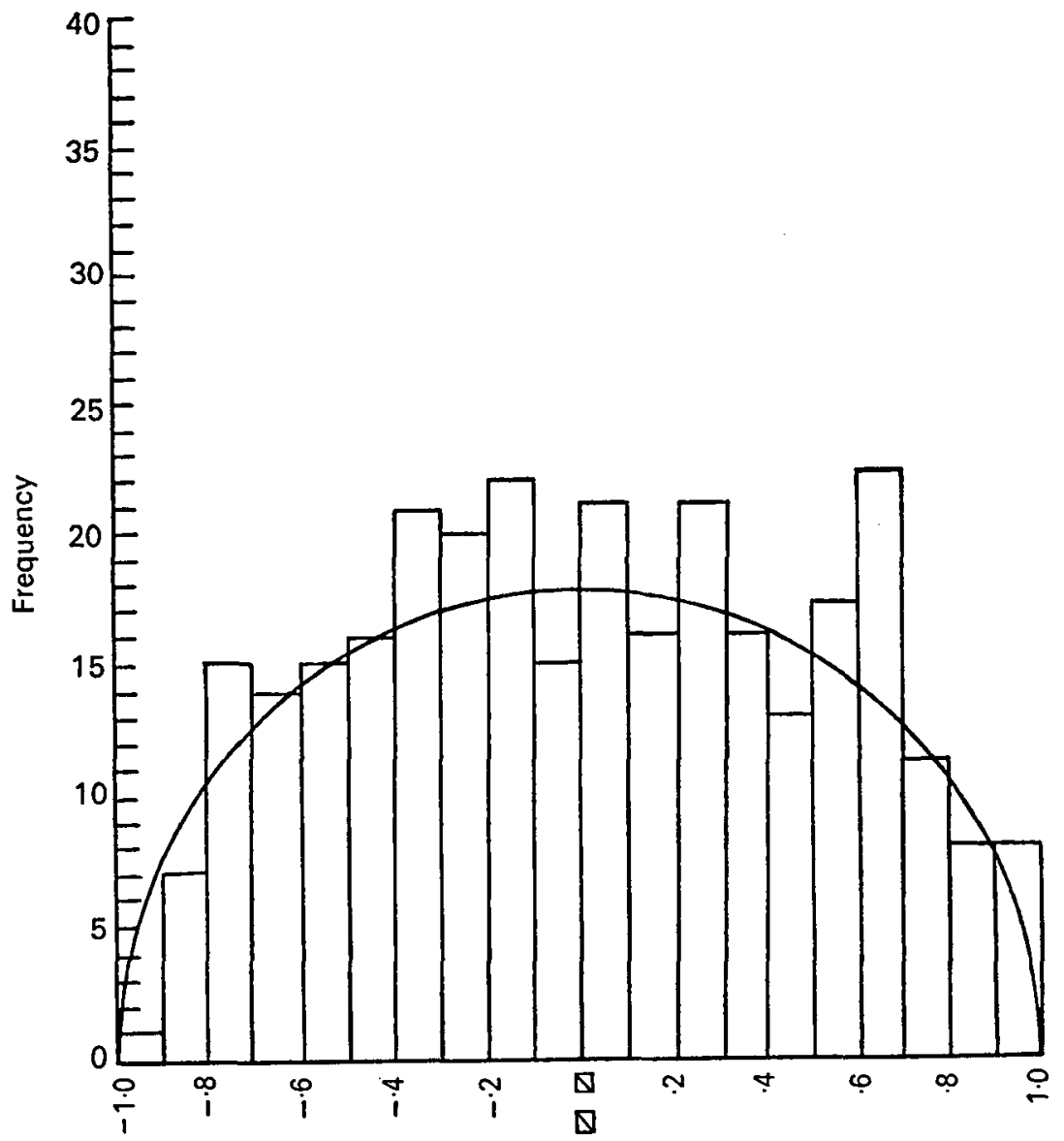

Histogram for $X_{(\pi)}$ and conjectured density function

FIGURe 2

The procedure used for calculating Table I actually produced all the $c(v)$ such that $\sigma(v)<60$, and in addition, certain values for $v$ with larger trace; the trace limitations were due to the limited precision of computation. The values which correspond to non prime $v$ make possible checks of the validity of the $c(\pi)$ for prime $\pi$ as a consequence of the following considerations. Because $\chi_{5}$ is an eigenfunction of the ring of Hecke operators associated with the Hilbert modular group for $\mathbb{Q}(\sqrt{ } 5)$, the results of Hermann [7] can be applied. Now Satz 11 of [7] can be interpreted in the following way in the present situation. Define the quartic character $\theta$ on the multiplicative subgroup of $\mathbb{Q}(\sqrt{ } 5)$ by the prescription

$$
\theta(\varepsilon)=\theta(\pi)=\theta\left(-\pi^{*}\right)=i
$$


if $\pi$ is an $\Xi^{*}$-reduced prime, and extend multiplicatively. Further define $c(\mu)$ for non totally positive $\mu / \sqrt{ } 5$ by $c(\varepsilon \mu)=\theta(\varepsilon) c(\mu)$. The eigenvalues of the Hecke operator $T(v)$ which corresponds to $v$ are just $\theta(v) a(v) / a(1)$, where $a(v)$ are the Fourier coefficients of eigenfunctions of the Hecke ring; since $c(\varepsilon)=1$ for the Fourier coefficient of $\chi_{5}$ (cf. Table I), it follows that $-i \theta(v) c(v)$ is the eigenvalue of $T(v)$. Then Satz 11 of [7] asserts that

$$
c(\mu) c(v)=\sum_{(\delta) \mid(\mu, v)} \bar{\theta}\left(\delta^{2} / \sqrt{ } 5\right)|\delta|^{4} c\left(\mu v / \delta^{2}\right)
$$

In particular, if $\mu$ and $v$ are relatively prime, then

$$
\begin{aligned}
c(\mu) c(v) & =\bar{\theta}\left(\frac{1}{\sqrt{ } 5}\right) c(\mu v) \\
& =+i c(\mu \nu) \\
& =-c(\varepsilon \mu \nu) .
\end{aligned}
$$

This multiplicative condition supplied a convenient test of the calculations which was applicable to all primes $\pi$ such that $2 \pi$ was within the range of our calculations. For instance, let $\pi$ be the $\Xi *$-reduced prime of absolute norm 1009; then Table I gives $c(\pi)=25870$, and $c(2 \varepsilon)=-10$. Our calculations showed that the $\Xi^{*}$-reduced integer of absolute norm $2^{2}(1009)=4036$ is $\mu=13+29 \sqrt{ } 5$ and $c(\mu)=-258700$. Since $\pi=\frac{13+29 \sqrt{ } 5}{2}=\frac{1}{2} \mu$, we find that $c(\mu)=c(2 \pi)=-c(\varepsilon(2 \varepsilon)(\pi))$ by $(9),=+c(2 \varepsilon) c(\pi)$, in agreement with $(13)$.

The more complicated instances of (12), wherein powers of a prime occur, can be applied to few of the tabulated entries because the norms of the powers rapidly grow beyond the bounds of the data. But there are several opportunities. For example, $\pi=1+2 \sqrt{ } 5$ is an $\Xi^{*}$-reduced prime and $a b s|\pi|=19$, while $\mu=\frac{1+17 \sqrt{ } 5}{2}=\varepsilon\left(\pi^{*}\right)^{2}$ is also reduced and evidently $a b s|\mu|=361$. Table I provides $c(\pi)=-140$ and the supplementary calculations show $c(\mu)=110721$. Application of $(12)$ yields

$$
\begin{aligned}
c(\pi)^{2} & =\theta\left(\frac{1}{\sqrt{ } 5}\right) c\left(\pi^{2}\right)+\theta\left(\frac{\pi^{2}}{\sqrt{ } 5}\right)|\pi|^{4} c(1) \\
& =i c\left(\pi^{2}\right)-i|\pi|^{4} c(1) \\
& =-c\left(\varepsilon \pi^{2}\right)+|\pi|^{4} c(\varepsilon) ;
\end{aligned}
$$

from (9) and (10), $c\left(\varepsilon \pi^{2}\right)=-c\left(-\varepsilon^{*} \pi^{* 2}\right)=+c\left(-\varepsilon^{2} \varepsilon^{*} \pi^{* 2}\right)=c\left(\varepsilon \pi^{* 2}\right)=c(\mu)$, and indeed, $(-140)^{2}=-110721+(19)^{4}$.

3. On calculating certain Fourier coefficients. A Hilbert modular form proportional to $\chi_{5}$ was first introduced by Gundlach [4] (cf. [11]) as the product of 10 theta functions associated with the principal congruence subgroup of level 2 of Hilbert's modular group for $\mathbb{Q}(\sqrt{ } 5)$. 
Although it would be possible to calculate the coefficients $c(v)$ directly from this definition, it is neither the simplest, nor an informative, way. Our efforts proceeded along a different path, thanks to an illuminating remark made to one of the authors (HLR) by A. Selberg in February 1973. Selberg remarked that automorphic forms in several complex variables give rise, in certain cases, to automorphic forms attached to submanifolds by restricting a type of normal derivative of the ambient form to the submanifold, and he adduced the following amusing example: let the product of classical upper half planes be embedded in the Siegel upper half plane of degree two as the diagonal matrices; the Cartesian product $\Gamma^{2}$ of the classical modular group with itself acts on the image as a subgroup of Siegel's modular group. If $z=\left(\begin{array}{ll}z_{1} & z_{3} \\ z_{3} & z_{2}\end{array}\right)$ be a point in the Siegel upper half plane and $\tilde{\phi}$ is a Siegel modular form of weight $w$, then the restriction $\phi=\left.\frac{\partial \Phi}{\partial z_{3}}\right|_{z_{3}=0}$ is a form of weight $w+1$ for $\Gamma^{2}$. If $w \equiv 0 \bmod 2$, then $\phi \equiv 0$, but if $w \equiv 1 \bmod 2$, then $\tilde{\phi}=\tilde{\psi} \tilde{\chi}_{35}$ where $\tilde{\psi}$ is a Siegel modular form of even weight and $\tilde{\chi}_{35}$ is a cusp form of weight 35. It follows that $\left.\frac{\partial \tilde{\phi}}{\partial z_{3}}\right|_{z_{3}=0}=\left.\left.\tilde{\psi}\right|_{z_{3}=0} \cdot \frac{\partial \tilde{\chi}_{35}}{\partial z_{3}}\right|_{z_{3}=0}$; the second factor is a skew cusp form of weight 36 for $\Gamma^{2}$ proportional, as one readily computes, to $\left\{\Delta\left(z_{1}\right) \Delta\left(z_{2}\right)\right\}^{2}\left\{g_{12}\left(z_{1}\right) \Delta\left(z_{2}\right)-g_{12}\left(z_{2}\right) \Delta\left(z_{1}\right)\right\}$, with $g_{w}\left(z_{k}\right)$ the Eisenstein series of weight $w$ and $\Delta\left(z_{k}\right)$ the (normalized) cusp form of weight 12 , both for the classical modular group.

The remainder of this section simply elaborates Selberg's remark in a restricted context which is nevertheless more general than that necessary for the applications described in this paper.

Let $\mathfrak{U}$ denote a compact real Jordan algebra of rank 2 with unit element $c$, reduced trace $a \mapsto \sigma(a)$ and reduced norm $a \mapsto|a|$ (cf. [1] for definitions and uncited results concerning Jordan algebras). Introduce $L(x)$ and $P(x)$ for $x \in \mathfrak{U}$ by $L(x) y=x y, P(x)=2 L(x)^{2}-L\left(x^{2}\right)$ for all $y \in \mathfrak{U}$, and extend these endomorphisms to $\mathfrak{U} \otimes \mathbb{C}$ in the natural way. $\mathscr{Z}(\mathfrak{U})=$ $\mathfrak{U}+i \exp \mathfrak{U}$ is biholomorphically equivalent to a bounded symmetric domain of rank 2 [8], where $\exp \mathfrak{U}=\left\{\exp a=\sum_{n=0}^{\infty} a^{n} / n !: a \in \mathfrak{U}\right\}$. Let $\Gamma$ be a discrete subgroup of the group Bih $\mathscr{Z}(\mathfrak{U})$ of biholomorphic automorphisms of $\mathscr{Z}(\mathfrak{U})$ and let $(\Gamma, w)$ denote the $\mathbb{C}$-linear space of holomorphic functions $\tilde{\phi}: \mathscr{Z}(\mathfrak{U}) \rightarrow \mathbb{C}$ such that

$$
\tilde{\phi}(\gamma z)=\operatorname{det}\left(\frac{\partial \gamma z}{\partial z}\right)^{-w / 2 q} \tilde{\phi}(z)
$$

where $\operatorname{det}\left(\frac{\partial \gamma z}{\partial z}\right)$ denotes the Jacobian determinant and $q=\operatorname{dim}_{\mathbb{R}} \mathfrak{U} /$ rank $\mathfrak{U}$. Let $\mathscr{M}_{a} \subset \mathscr{Z}(\mathfrak{U})$ be the linear complex submanifold of codimension 1 in $\mathscr{Z}(\mathfrak{U})$ defined by

$$
\mathscr{M}_{a}=\{z \in \mathscr{Z}(\mathfrak{U}): \sigma(a z)=0\}
$$

for a fixed $a \in \mathfrak{U}-\{0\}$, and let $\Gamma_{a} \subset \Gamma$ be the subgroup of biholomorphic automorphisms of $\mathscr{Z}(\mathfrak{U})$ which preserve $\mathscr{M}_{a}$. The restriction of $\operatorname{det}\left(\frac{\partial \gamma z}{\partial z}\right)^{-w / 2 q}$ to $\mathscr{M}_{a}$ defines a factor of auto- 
morphy on $\mathscr{H}_{a}$, and we denote the $\mathbb{C}$-linear space of $\Gamma_{a}$-automorphic forms relative to this factor of automorphy by $\left(\Gamma_{a}, w\right)$. Evidently $\tilde{\phi} \in(\Gamma, w)$ implies that $\tilde{\phi} \mid \mathscr{M}_{a} \in\left(\Gamma_{a}, w\right)$.

Introduce the $\sigma$-gradient $\nabla$ by

$$
\frac{\partial f}{\partial z}(b)=\sigma\left(b, \nabla_{z} f\right)=\sigma(b, \nabla f), \quad b \in \mathfrak{U} \otimes \mathbb{C},
$$

for differentiable $f: \mathscr{Z}(\mathfrak{U}) \rightarrow \mathbb{C} ; \sigma(a, \nabla f)$ is the gradient of $f$ in a direction normal to $\mathscr{M}_{a}$. We will investigate the restriction $\left.\sigma(a, \nabla \tilde{\phi})\right|_{\mu_{a}}$ for $\tilde{\phi} \in(\Gamma, w)$. Recalling [8] that $\operatorname{Bih} \mathscr{Z}(\mathfrak{U})$ is generated by maps of the form

$$
\left\{\begin{array}{l}
z \mapsto z+t, \quad t \in \mathfrak{U}, \\
z \mapsto A z, \quad A \in \text { Aut } \mathfrak{U}=\text { automorphisms of } \mathfrak{U}, \\
z \mapsto-z^{-1}
\end{array}\right.
$$

we find that

$$
\sigma(a, \nabla \tilde{\phi})(z+t)=\sigma(a, \nabla \tilde{\phi})(z)
$$

Further,

$$
\sigma(a, \nabla \tilde{\phi})(A z)=\sigma\left(a, \nabla_{A z} \tilde{\phi}\right)(z) ;
$$

now use $\nabla_{A z}=A^{-1} \nabla_{z}$ and the fact that automorphisms are self-adjoint with respect to $\sigma$ to obtain

$$
=\sigma\left(A^{-1} a, \nabla_{z} \delta\right)(z) .
$$

Last, from $\nabla_{-z^{-1}}=P(z) \nabla_{z}$ and $\phi\left(-z^{-1}\right)=|z|^{w} \phi(z)$, we find that

$$
\begin{gathered}
\sigma(a, \nabla \tilde{\phi})\left(-z^{-1}\right)=\sigma\left(a, P(z) \nabla_{z}|z|^{w} \tilde{\phi}(z)\right) \\
=|z|^{w} \sigma\left(a, P(z) \nabla_{z} \tilde{\phi}\right)(z)+\tilde{\phi}(z) \sigma\left(a, P(z) \nabla|z|^{w}\right) .
\end{gathered}
$$

One readily verifies that

$$
\nabla|z|^{w}=w|z|^{w} z^{-1}
$$

recalling that $P(z)$ is self-adjoint with respect to $\sigma,(19)$ becomes

$$
\sigma(a, \nabla \tilde{\phi})\left(-z^{-1}\right)=|z|^{w} \sigma(P(z) a, \nabla \tilde{\phi})(z)+w|z|^{w} \tilde{\phi}(z) \sigma(a, z) .
$$

Now restrict $z$ in (19), (18), and (20) to $\mathscr{M}_{a}$ and $A$ to Aut $\mathfrak{U} \cap \Gamma_{a}$. Then $z \in \mathscr{M}_{a}$ implies that $A^{-1} z \in \mathscr{M}_{a}$; so $0=\sigma\left(a, A^{-1} z\right)=\sigma\left(A^{-1} a, z\right)$, from which we conclude that $A^{-1} a=a$. The restriction of (20) is

$$
\left.\sigma(a, \nabla \tilde{\phi})\left(-z^{-1}\right)\right|_{\mathscr{M}_{a}}=\left.|z|^{w} \sigma(P(z) a, \nabla \tilde{\phi})(z)\right|_{\mathcal{M}_{a}},
$$


where we assume that $z \leftrightarrow-z^{-1} \in \Gamma_{a}$. These relations show that $\left.\sigma(a, \nabla \tilde{\varphi})\right|_{\mathscr{A}_{a}}$ will be a $\Gamma_{a^{-}}$ automorphic form if and only if there is a function $\lambda: \mathfrak{H} \otimes \mathbb{C} \rightarrow \mathbb{C}$ such that

$$
\sigma(P(z) a, \nabla \tilde{\phi})(z)=\lambda(z) \sigma(a, \nabla \tilde{\phi})(z) .
$$

At this point we use the rank restriction on $\mathfrak{U}$ : if $u \in \mathfrak{U} \otimes \mathbb{C}$, then

$$
u^{2}-\sigma(u) u+|u| c=0 .
$$

Let $a^{\mathfrak{t}}$ denote a square root of $a \in \mathfrak{U}-\{0\}$ and set $u=P\left(a^{\frac{1}{2}}\right) z$. Then substitution in (23) produces

$$
\left(P\left(a^{\frac{1}{4}}\right) z\right)^{2}-\sigma\left(P\left(a^{\frac{1}{2}}\right) z\right) P\left(a^{\frac{1}{2}}\right) z+\left|P\left(a^{\frac{1}{4}}\right) z\right|=0 ;
$$

now use the well-known properties [1]:

to conclude that

$$
\begin{aligned}
u^{2} & =P(u) c, \\
P(P(u) v) & =P(u) P(v) P(u), \quad P^{n}(u)=P\left(u^{n}\right), \\
\left|P\left(u^{\frac{1}{2}}\right) v\right| & =|u||v|
\end{aligned}
$$

$$
P\left(a^{\frac{1}{2}}\right) P(z) P\left(a^{\frac{1}{3}}\right) c-\sigma(a, z) P\left(a^{\frac{1}{3}}\right) z+|a||z| c=0 .
$$

Restriction of (24) to $\mathscr{M}_{a}$ yields

$$
P(z) a=-|a||z| P^{-1}\left(a^{\frac{1}{2}}\right) c=-|a||z| a^{-1},
$$

and substitution of (25) in (22) provides the equality

$$
\begin{aligned}
& \left.\sigma(P(z) a, \nabla \delta)\right|_{\mathcal{M}_{a}} \\
& \| \\
& -\left.|a||z| \sigma\left(a^{-1}, \nabla \delta\right)\right|_{\mathcal{H}_{a}}
\end{aligned}
$$

Hence, $\left.\sigma(a, \nabla \phi)\right|_{\mathcal{H}_{a}}$ will be an automorphic form if $a^{-1}=\mu a$ for some $\mu \in \mathbb{R}$, that is

We have proved

$$
a^{2} \in \mathbb{R} c \text {. }
$$

THEOREM 1. If $a^{2} \in \mathbb{R} c$ and $a \neq 0$, then

$$
\left.\sigma(a, \nabla)\right|_{\mathcal{M}_{a}}:\left(\Gamma_{a}, w\right) \rightarrow\left(\Gamma_{a}, w+1\right) .
$$

The theorem can be applied to Hammond's embedding [6] of certain Hilbert modular groups in Siegel's modular group of degree two. Let $k$ denote a real quadratic number field with discriminant $\Delta$ which is a sum of two squares:

$$
\Delta=u^{2}+v^{2}, v \equiv 0 \bmod 2 .
$$


Let $\Gamma_{\Delta}$ denote the corresponding Hilbert modular group acting on the product $\mathscr{H}$ of halfplanes in the usual way, and write $\zeta=\left(\zeta_{1}, \zeta_{2}\right) \in \mathscr{H}$ as in $\S 1$. Put

$$
\eta_{1}=\frac{u+\sqrt{ } \Delta}{2}, \eta_{2}=\frac{u-\sqrt{ } \Delta}{2} \text {. }
$$

The map

$$
\begin{aligned}
& \sqrt{\Delta} z_{1}=\eta_{1} \zeta_{1}-\eta_{2} \zeta_{2} \\
& \sqrt{\Delta} z_{2}=-\eta_{2} \zeta_{1}+\eta_{1} \zeta_{2} \\
& \sqrt{\Delta} z_{3}=+\sqrt{-|\eta|}\left(\zeta_{1}-\zeta_{2}\right)
\end{aligned}
$$

embeds $\mathscr{H}$ onto the submanifold of codimension 1 in Siegel's upper half plane $\mathscr{Z}$ of degree two which is given by

$$
\mathscr{M}_{u, v}=\left\{z \in \mathscr{Z}: \frac{v}{2}\left(z_{1}-z_{2}\right)-u z_{3}=0\right\} .
$$

Identify $\mathscr{Z}$ with the half space $\mathscr{Z}(\mathfrak{U})$ corresponding to the Jordan algebra $\mathfrak{U}$ of $2 \times 2$ symmetric real matrices; then $\sigma$ denotes matrix trace and $|\cdot|$ denotes the determinant of a matrix. Set

then (30) can be written

$$
a=\frac{1}{2}\left(\begin{array}{rr}
v & -u \\
-u & -v
\end{array}\right)
$$

$$
\mathscr{M}_{u, 0}=\{z \in \mathscr{Z}(\mathfrak{U}): \sigma(a z)=0\} .
$$

Observe that $a^{2}=-\frac{\Delta}{4}\left(\begin{array}{ll}1 & 0 \\ 0 & 1\end{array}\right)$; hence the theorem can be applied. It is known [6] that the embedding (29) induces an isomorphism of $\Gamma_{\Delta}$ into a subgroup of Siegel's modular group of degree two $\Gamma$. Write $\partial_{k}=\frac{\partial}{\partial z_{k}}$; then we have

THeOREM 2. If $\tilde{\phi} \in(\Gamma, w)$, then

$$
\left.\phi \equiv\left\{\frac{v}{2}\left(\partial_{1}-\partial_{2}\right)-\frac{u}{2} \partial_{3}\right\} \hat{\phi}\right|_{\mathcal{H}_{u, v}} \in\left(\Gamma_{\Delta}, w+1\right) .
$$

Moreover, $\phi$ is skew, i.e.

$$
\phi\left(\zeta_{1}, \zeta_{2}\right)=-\phi\left(\zeta_{2}, \zeta_{1}\right)
$$

Only the last assertion has not been proved. But note that

$$
\begin{aligned}
& z \mapsto\left(\begin{array}{rr}
0 & 1 \\
-1 & 0
\end{array}\right) z\left(\begin{array}{rr}
0 & -1 \\
1 & 0
\end{array}\right)=\left(\begin{array}{rr}
z_{2} & -z_{3} \\
-z_{3} & z_{1}
\end{array}\right)=z^{\#} \in \Gamma \text { and } \\
& \tilde{\phi}\left(z^{\#}\right)=\tilde{\phi}(z) ; \text { hence } \phi\left(\zeta_{1}, \zeta_{2}\right)=\left.\tilde{\phi}(z)\right|_{\mathcal{A}_{u, v}}=\left.\tilde{\phi}\left(z^{\#}\right)\right|_{\mathcal{A}_{u, v}}=\phi\left(\zeta_{2}, \zeta_{1}\right)
\end{aligned}
$$


by (29), whereas the differential operator $\sigma(a \nabla)$ changes sign, under $z \mapsto z^{\#}$. This completes the proof.

Let the Fourier expansion of $\tilde{\phi} \in(\Gamma, w)$ be

$$
\tilde{\phi}(z)=\sum_{n \geqq 0} \tilde{c}(n) e^{2 \pi i \sigma(n z)}
$$

here the sum runs over all semi-integral semi-positive definite real symmetric matrices. Let

$$
\phi(\zeta)=\left.\sigma(a, \nabla \tilde{\phi})(z)\right|_{\mathcal{M}_{u, v}}
$$

with $a$ given by (31), and let the Fourier expansion of $\phi$ be

$$
\phi(\zeta)=2 \pi i \sum_{v / \sqrt{ } \Delta \geqq 0} c(v) \exp 2 \pi i \sigma\left(\frac{v \zeta}{\sqrt{ } \Delta}\right),
$$

where $v$ runs through the integral elements of $k$ such that $v / \sqrt{ } \Delta$ is 0 or totally positive. Then comparison of (33) with (34) yields

THEOREM 3.

(a) $c(v)=\sum_{n \geqq 0}\left\{\frac{v}{2}\left(n_{1}-n_{2}\right)-u n_{3}\right\} \tilde{c}(n)$ $n_{3} v+n_{1} \eta_{1}-n_{2} \eta_{2}=v$ where $v$ runs through the integral elements of $k$ such that $v / \sqrt{ } \Delta>0$;

(b) $c(0)=0$;

(c) $c(\sqrt{ } \Delta)=0$.

COROLlaRY 4. If the discriminant $\Delta$ of a quadratic number field is a sum of two squares, then there is a skew non-identically zero $\Gamma_{\Delta^{-c}}$-usp form of weight $w$ for every odd $w \geqq 5$.

Proof. There is an Eisenstein series $\tilde{\phi}_{\tilde{w}}$ of weight $\tilde{w} \equiv 0 \bmod 2$ for $\tilde{w} \geqq 4$; the corresponding $\phi_{w}$ is a skew cusp form of weight $w=\tilde{w}+1 . \phi_{w}$ is not identically zero because

$$
c\left(\frac{u+\sqrt{ } \Delta}{2}\right)=c\left(\eta_{1}\right)=\frac{v}{2} \tilde{c}\left(\begin{array}{ll}
1 & 0 \\
0 & 0
\end{array}\right),
$$

but $\tilde{c}\left(\begin{array}{ll}1 & 0 \\ 0 & 0\end{array}\right)=(-)^{w / 2} \frac{2 w}{B_{w / 2}}$, where $B_{w / 2}$ is the Bernoulli number, $\neq 0$; this latter assertion is valid because the Siegel $\Phi$-operator carries Eisenstein series to Eisenstein series of the same weight. 

to be

If $u=v / 2=1$, then $\Delta=5$ and the normalized cusp form $\chi_{5}$ introduced in (4) turns out

$$
\left.\frac{1}{2 \pi i} \cdot \frac{1}{240} \sigma\left(a, \nabla \tilde{\phi}_{4}\right)\right|_{\mathscr{M}_{1,2}}=\chi_{5}
$$

where $\phi_{4} \in(\Gamma, 4)$ is the normalized Eisenstein series of weight 4 and $a=\frac{1}{2}\left(\begin{array}{rr}2 & -1 \\ -1 & -2\end{array}\right)$; thus, knowledge of the Fourier coefficients of $\tilde{\phi}_{4}$ enables one to calculate the Fourier coefficients of $\chi_{s}$ by using Theorem 3 . The entries in Table $I$ were calculated in this manner, making use of the linear recursions given in [12] to calculate the coefficients of $\bar{\phi}_{4}$.

It is evident from Theorem 3 that the values of the Fourier coefficients of $\tilde{\phi}_{4}$ also enable one to calculate the coefficients of a non-identically zero skew cusp form in $\left(\Gamma_{\Delta}, 5\right)$, but it is not known whether this form spans the space $\left(\Gamma_{\Delta}, 5\right)$ except for $\Delta=5,8$, in which cases the answer is affirmative [4], [5], [12]. Nevertheless, it may be of some interest to tabulate some of these coefficients for fields with class number greater than 1 . This question is taken up in $\S 4$ below.

Equation (36) shows that the sum in Theorem 3 telescopes to one term if $v=(u+\sqrt{ } \Delta) / 2$. This example is a special case of a general identity theorem which it may not be out of place to include here. Set $\mathscr{D}(r)=\{x \in \mathfrak{U}: \sigma(x) \leqq r\} \cap \exp \mathfrak{U} ; \mathscr{D}(r)$ is a closed disk. The semiintegral matrices $n \geqq 0$ such that $\sigma(n)=r$ constitute the intersection of a lattice in $\mathfrak{U}$ with $\mathscr{D}(r)$. If we put $\mathfrak{U} \in X=\left(\begin{array}{ll}x_{1} & x_{3} \\ x_{3} & x_{2}\end{array}\right), X=x_{1}-x_{2}, Y=2 x_{3}$, then $\mathscr{D}(r)=\left\{x:\left(x_{1}-x_{2}\right)^{2}+\right.$ $\left.4 x_{3}^{2} \leqq r^{2}=\left(x_{1}+x_{2}\right)^{2}\right\}$ and the semi-integral matrices $n \geqq 0$ in $\mathscr{D}(r)$ correspond to coordinate pairs $(X, Y) \in \mathbb{Z}^{2}$ such that $X \equiv r(\bmod 2)$ and $X^{2}+Y^{2} \leqq r^{2}$. Moreover, we find from (30) that $\mathscr{D}(r) \cap \mathscr{M}_{u, v}=\{x \in \mathscr{D}(r): v X-u Y=0\}$. Now suppose that $\delta \in(\Gamma, w)$; then $\left.\tilde{\phi}\right|_{\mathcal{M}_{u, v}} \in\left(\Gamma_{\Delta}, w\right)$ and the Fourier coefficients $c(v)$ of $\left.\tilde{\phi}\right|_{\mathcal{M}_{u, v}}$ can be expressed in terms of the coefficients $\tilde{c}(n)$ of $\tilde{\phi}$ by the formula

$$
\begin{aligned}
c(v)= & \sum_{n \geqq 0} \tilde{c}(n) \\
& n_{3} v+n_{1} \eta_{1}-n_{2} \eta_{2}=v
\end{aligned}
$$

(cf. the analogous formula in Theorem 3). The condition $n_{3} v+n_{1} \eta_{1}-n_{2} \eta_{2}=v$ is equivalent to the pair

and

$$
\sigma(n)=\sigma(v / \sqrt{ } \Delta)
$$

$$
u M+v N=v+v^{*}
$$

where $M=n_{1}-n_{2}, N=2 n_{3}$, and $v^{*}$ is the conjugate of $v$. The lattice points $(M, N)$ in $\mathscr{D}(r)$ consequently lie on a line perpendicular to $\mathscr{D}(r) \cap \mathscr{M}_{u, v}$. It follows that if $n$ is fixed, then for all but a finite number of discriminants $\Delta$, the line through $n$ perpendicular to $\mathscr{M}_{u, v}$ will not contain any other semi-integral matrix $n \geqq 0$ and therefore

$$
c(v)=\tilde{c}(n) \text { with } v=n_{3} v+n_{1} \eta_{1}-n_{2} \eta_{2}
$$

for all but a finite number of discriminants. This observation leads to 
THEOREM 5. There is an integer $K$ depending only on $w$ such that if $\tilde{\phi}, \tilde{\psi} \in(\Gamma, w)$ and $c(v)$ are the Fourier coefficients of $\left.(\tilde{\phi}-\tilde{\psi})\right|_{\mathcal{M}_{\tilde{u}}, v}$, then, for all but a finite number of discriminants, $c(v)=0$ for $\sigma(v / \sqrt{ } \Delta)<$ Kimplies that $\tilde{\phi} \equiv \tilde{\psi}$.

Proof. $\operatorname{dim}_{\mathbb{C}}(\Gamma, w)<\infty$ implies the existence of a $K$ which depends only on $w$ such that $\tilde{c}(n)=0$ for $\sigma(n)<K$ gives $\tilde{\phi} \equiv 0$, where the $\tilde{c}(n)$ are the Fourier coefficients of $\tilde{\phi} \in(\Gamma, w)$. Let $\mathscr{S}_{K}$ denote the set of slopes of the lines determined by pairs of semi-integral matrices $n^{\prime} \geqq 0, n^{\prime \prime} \geqq 0$ such that $\sigma\left(n^{\prime}\right)=\sigma\left(n^{\prime \prime}\right)<K$. If $\Delta=u^{2}+v^{2}$ is the discriminant of the field generated by a positive square free integer, then $u$ and $v$ can have at most the factor 2 in common and therefore the set of slopes of the line segments $\left\{\mathscr{D}(r) \cap \mathscr{M}_{u, v}: \Delta=u^{2}+v^{2}\right.$ is a discriminant and $v \equiv 0(\bmod 2)\}$ is the set of distinct numbers $\left\{v /(2 u): u^{2}+v^{2}\right.$ is a discriminant and $v \equiv 0(\bmod 2)\}$. Hence, for all but a finite number of discriminants, $v /(2 u) \notin \mathscr{S}_{K}$ and consequently (39) is valid for $\sigma(n)<K$. It follows that $c(v)=0$ for $\sigma(v / \sqrt{ } \Delta)<K$ implies that $\tilde{\phi} \equiv 0$. Now replace $\tilde{\phi}$ by $(\tilde{\phi}-\tilde{\psi})$ to obtain the statement of the Theorem.

Let $c(v)$ denote the $v$ th Fourier coefficient of $\phi=\left.\sigma(a, \nabla \delta)\right|_{\mathcal{A}_{u, v}}, a=\frac{1}{2}\left(\begin{array}{rr}v & -u \\ -u & -v\end{array}\right)$. A similar argument shows that there is a constant $K$ such that $c(v)=0$ for $\sigma(v / \sqrt{ } \Delta)<K$ implies

table III. Fourier Coefficients of the Normalized Cusp Form $\chi_{5}$ for Hilbert's Modular Group AsSociated WITH $\mathbb{Q}(\sqrt{ } 10)$ For REDUCED $v / \sqrt{ } 40$. $v=k+l(20+\sqrt{ } 10)$.

\begin{tabular}{|c|c|c|c|c|c|c|c|}
\hline$a b s|v|$ & $l$ & $-k$ & $c(v)$ & $a b s|v|$ & $l$ & $-k$ & $c(v)$ \\
\hline 1 & 1 & 17 & 1 & 186 & 5 & 92 & 24192 \\
\hline 4 & $\frac{1}{2}$ & 34 & 18 & 201 & 5 & 93 & 10332 \\
\hline 36 & 2 & 38 & -6 & 214 & 5 & 94 & -25344 \\
\hline $\begin{array}{l}39 \\
40\end{array}$ & $\begin{array}{l}2 \\
2\end{array}$ & $\begin{array}{l}39 \\
40\end{array}$ & $\begin{array}{r}-168 \\
0\end{array}$ & $\begin{array}{l}225 \\
234\end{array}$ & $\begin{array}{l}5 \\
5\end{array}$ & $\begin{array}{l}95 \\
96\end{array}$ & $\begin{array}{l}-37785 \\
-24192\end{array}$ \\
\hline 9 & 3 & 51 & 84 & 241 & 5 & 97 & 11592 \\
\hline 65 & 3 & 55 & -630 & 246 & 5 & 98 & 48384 \\
\hline 74 & 3 & 56 & -1152 & 249 & 5 & 99 & 43092 \\
\hline 81 & 3 & $\begin{array}{l}57 \\
58\end{array}$ & 756 & 250 & 5 & 100 & 0 \\
\hline $\begin{array}{l}86 \\
89\end{array}$ & $\begin{array}{l}3 \\
3\end{array}$ & $\begin{array}{l}58 \\
59\end{array}$ & $\begin{array}{r}2304 \\
882\end{array}$ & $\begin{array}{r}36 \\
104\end{array}$ & 6 & $\begin{array}{l}102 \\
104\end{array}$ & $\begin{array}{r}252 \\
-1008\end{array}$ \\
\hline 16 & 4 & 68 & $\begin{array}{l}002 \\
292\end{array}$ & 135 & $\begin{array}{l}0 \\
6\end{array}$ & 105 & -7560 \\
\hline 79 & 4 & 71 & -392 & 164 & 6 & 106 & -8316 \\
\hline 96 & 4 & 72 & -3024 & 191 & 6 & 107 & 5544 \\
\hline 111 & 4 & 73 & -1512 & 216 & 6 & 108 & 30240 \\
\hline 124 & 4 & 74 & 4144 & 239 & 6 & 109 & 31320 \\
\hline 135 & 4 & 75 & 7560 & 260 & 6 & 110 & -10080 \\
\hline 144 & 4 & 76 & 5940 & 279 & 6 & 111 & -65016 \\
\hline 151 & 4 & 77 & -4568 & 296 & 6 & 112 & -83232 \\
\hline 156 & 4 & 78 & -15120 & 311 & 6 & 113 & -24192 \\
\hline 159 & 4 & 79 & -12096 & 324 & 6 & 114 & 61488 \\
\hline 160 & 4 & 80 & 0 & 335 & 6 & 115 & 98280 \\
\hline 25 & $\begin{array}{l}4 \\
5\end{array}$ & 85 & 630 & 344 & 6 & 116 & 79776 \\
\hline 106 & 5 & 88 & -3456 & 351 & 6 & 117 & -3528 \\
\hline 129 & 5 & 89 & -6216 & 356 & 6 & 118 & -77364 \\
\hline 150 & 5 & 90 & & 359 & 6 & 119 & -71856 \\
\hline 169 & 5 & 91 & 12474 & 360 & 6 & 120 & \\
\hline
\end{tabular}


that $\tilde{\phi}=0$. Indeed, according to Theorem 3 and the argument given above, for all but finitely many discriminants, $\sigma(v / \sqrt{ } \Delta)<K$ implies that

$$
c(v)=\left\{\frac{v}{2}\left(n_{1}-n_{2}\right)-u n_{3}\right\} \tilde{c}(n)
$$

with $v=n_{3} v+n_{1} \eta_{1}-n_{2} \eta_{2}$. Thus $c(v)=0$ but $\tilde{c}(n) \neq 0$ implies that $(v / 2)\left(n_{1}-n_{2}\right)=u n_{3}$ in this range. But one readily proves that a Siegel modular form cannot have all its non-zero Fourier coefficients $\tilde{c}(n), \sigma(n)<K$ supported on a linear submanifold of positive codimension.

4. Applications with $\boldsymbol{\Delta}=\mathbf{4 0}$ and $\boldsymbol{\Delta}=\mathbf{2 2 9}$. In this section Theorem $\mathbf{3}$ is applied to obtain the leading Fourier coefficients of $\left.\chi_{5} \equiv \sigma\left(a, \nabla \tilde{\phi}_{4}\right)\right|_{\mathcal{H}_{u, v}}$ for $\Delta=40$ and $\Delta=229$. These cases are of special interest because the class number of the corresponding fields are greater

TABle IV. Fourier Coefficients of the Normalized Cusp Form $\chi_{5}$ for Hilbert's MODUlar Group ASSOCIATED WITH Q(229 $)$ FOR REDUCED $v / \sqrt{ } 229$.

\begin{tabular}{|c|c|c|c|c|c|c|c|}
\hline$a b s|v|$ & $l$ & $-k$ & $c(v)$ & $a b s|v|$ & $l$ & $-k$ & $c(v)$ \\
\hline $\begin{array}{r}1 \\
4 \\
225 \\
228 \\
229 \\
9 \\
425 \\
443 \\
459 \\
473 \\
485 \\
16 \\
592 \\
627 \\
660 \\
691 \\
720 \\
747 \\
772 \\
900 \\
907 \\
912 \\
915 \\
916 \\
25 \\
729 \\
781 \\
831 \\
879 \\
925 \\
969 \\
1011 \\
1051 \\
1089 \\
1299\end{array}$ & $\begin{array}{l}1 \\
2 \\
2 \\
2 \\
2 \\
3 \\
3 \\
3 \\
3 \\
3 \\
3 \\
4 \\
4 \\
4 \\
4 \\
4 \\
4 \\
4 \\
4 \\
4 \\
4 \\
4 \\
4 \\
4 \\
5 \\
5 \\
5 \\
5 \\
5 \\
5 \\
5 \\
5 \\
5 \\
5 \\
5\end{array}$ & $\begin{array}{l}107 \\
214 \\
227 \\
228 \\
229 \\
321 \\
334 \\
335 \\
336 \\
337 \\
338 \\
428 \\
440 \\
441 \\
442 \\
443 \\
444 \\
445 \\
446 \\
454 \\
455 \\
456 \\
457 \\
458 \\
535 \\
546 \\
547 \\
548 \\
549 \\
550 \\
551 \\
552 \\
553 \\
554 \\
561\end{array}$ & $\begin{array}{r}1 \\
18 \\
-15 \\
-420 \\
0 \\
84 \\
-1764 \\
-3744 \\
756 \\
4896 \\
2016 \\
292 \\
-1148 \\
-9828 \\
-8316 \\
4144 \\
14364 \\
12852 \\
1372 \\
-270 \\
-12960 \\
-37800 \\
-30240 \\
0 \\
630 \\
-27 \\
-11232 \\
-24864 \\
-18144 \\
12474 \\
42336 \\
37296 \\
14688 \\
33 \\
-21924\end{array}$ & $\begin{array}{r}1321 \\
1341 \\
1359 \\
1375 \\
1389 \\
1401 \\
1411 \\
1419 \\
36 \\
905 \\
972 \\
1037 \\
1100 \\
1161 \\
1220 \\
1277 \\
1332 \\
1385 \\
1661 \\
1700 \\
1737 \\
1772 \\
1805 \\
1836 \\
1865 \\
1892 \\
1917 \\
1940 \\
1961 \\
2025 \\
2036 \\
2045 \\
2052 \\
2057 \\
2060 \\
2061\end{array}$ & $\begin{array}{l}5 \\
5 \\
5 \\
5 \\
5 \\
5 \\
5 \\
5 \\
6 \\
6 \\
6 \\
6 \\
6 \\
6 \\
6 \\
6 \\
6 \\
6 \\
6 \\
6 \\
6 \\
6 \\
6 \\
6 \\
6 \\
6 \\
6 \\
6 \\
6 \\
6 \\
6 \\
6 \\
6 \\
6 \\
6 \\
6\end{array}$ & $\begin{array}{l}562 \\
563 \\
564 \\
565 \\
566 \\
567 \\
568 \\
569 \\
642 \\
653 \\
654 \\
655 \\
656 \\
657 \\
658 \\
659 \\
660 \\
661 \\
667 \\
668 \\
669 \\
670 \\
671 \\
672 \\
673 \\
674 \\
675 \\
676 \\
677 \\
681 \\
682 \\
683 \\
684 \\
685 \\
686 \\
687\end{array}$ & $\begin{array}{r}-86688 \\
-105840 \\
-78624 \\
11592 \\
102816 \\
120960 \\
94752 \\
23436 \\
1512 \\
-3276 \\
-27972 \\
-45738 \\
-19404 \\
30240 \\
63756 \\
79002 \\
40068 \\
4284 \\
-20448 \\
-144648 \\
-247968 \\
-277056 \\
-133056 \\
61992 \\
229824 \\
362304 \\
296352 \\
165312 \\
22752 \\
-1260 \\
-56700 \\
-226800 \\
-341460 \\
-374220 \\
-204120 \\
0\end{array}$ \\
\hline
\end{tabular}


than 1. In fact, 40 is the least discriminant corresponding to a field of class number 2 which is a sum of two squares, and 229 is the least discriminant of a field of class number 3 which is prime and a sum of two squares. It is not known whether the Hilbert modular form $\chi_{5}$ is an eigenfunction of the Hecke ring in either of these cases.

A basis for the ring of integers of the field $k_{\Delta}$ of discriminant $\Delta$ is, in any case, given by 1 and $\omega=(\Delta+\sqrt{ } \Delta) / 2$. If $v=k+l \omega$, then

$$
\sigma\left(\frac{v}{\sqrt{ } \Delta}\right)=l
$$

Tables III and IV above present some Fourier coefficients $c(v)$ of $\chi_{5}$ for $\Delta=40$ and $\Delta=229$ respectively. In both cases, $v$ is constrained to lie in the domain $\left\{\alpha:-1 \geqq \frac{\alpha_{1}}{\alpha_{2}} \geqq \frac{\eta_{1}}{\eta_{2}}\right\}$, where $\eta_{1}=(u+\sqrt{ } \Delta) / 2$, and $(u, v)=(6,4)$ for $\Delta=40$ and $(u, v)=(15,4)$ for $\Delta=229$. Note that $\eta_{1}$ is a fundamental unit in each case.

\section{REFERENCES}

1. H. Braun and M. Koecher, Jordan-Algebren, Springer, Berlin, 1966. No. 355

2. P. Deligne, Formes modulaires et représentations $l$-adiques, Sém. Bourbaki 21 (1968/69),

3. P. Deligne, La conjecture de Weil. I, Publ. Math. IHES No. 43, to appear.

4. K.-B. Gundlach, Die Bestimmung der Funktionen zur Hilbertschen Modulgruppe des Zahlkörpers $\mathbb{Q}(\sqrt{ } 5)$, Math. Ann. $152(1963), 226-256$.

5. K.-B. Gundlach, Die Bestimmung der Funktionen zu einigen Hilbertschen Modulgruppen, J. fiir die reine und angew. Math. 220 (1965), 109-153. 497-516.

6. W. F. Hammond, The modular groups of Hilbert and Siegel, Amer. J. Math. 88 (1966),

7. O. Hermann, Über Hilbertsche Modulfunktionen und die Dirichletschen Reihen mit Eulerscher Produktentwicklung, Math. Ann. 127 (1954), 357-400.

8. M. Koecher, An Elementary Approach to Bounded Symmetric Domains (Rice University, Houston, 1969).

9. D. H. Lehmer, Note on the distribution of Ramanujan's tau function, Math. Computation 24 (1970), 741-743. 159-184.

10. S. Ramanujan, On certain arithmetical functions, Trans. Cambridge Phil. Soc. 22 (1916),

11. H. L. Resnikoff, On the graded ring of Hilbert modular forms associated with $\mathbb{Q}(\sqrt{ } 5)$, Math. Ann. 208 (1974), 161-170.

12. H. L. Resnikoff and R. L. Saldaña, Some properties of Fourier coefficients of Eisenstein series of degree two, J. für die reine und angew. Math. 265 (1974), 90-109.

13. J.-P. Serre, Une interprétation des congruences relatives à la fonction $\tau$ de Ramanujan, Sém. Delange-Pisot-Poitou $9(1967 / 68)$, No. 14. 1968).

14. J.-P. Serre, Abelian l-adic Representations and Elliptic Curves (W. A. Benjamin, New York,

15. J.-P. Serre, Congruences et formes modulaires, Sém. Bourbaki 24 (1971/72), No. 416.

16. J. T. Tate, Algebraic cycles and poles of zeta functions, in Arithmetical Algebraic Geometry (Harper and Row, New York, 1965).

RICE UNIVERSITY

Houston, TeXas 77001

U.S.A.
Present address:

UNIVERSity OF CALIFORNIA

IRVINE

CALIFORNIA 92664, U.S.A. 